\title{
Melanocytoma of the Eyeball
}

National Cancer Institute

\section{Source}

National Cancer Institute. Melanocytoma of the Eyeball. NCI Thesaurus. Code C4230.

A benign melanocytic proliferation within or adjacent to the optic disk. It presents as a pigmented, intraocular tumor. 